\title{
Desempenho de indicadores de obesidade abdominal e risco cardiovascular de idosos atendidos na rede básica de saúde do município de Belém - PA
}

Rodolfo Gomes do Nascimento*, Zeneide Nazaré Lima dos Santos", Ronald de Oliveira Cardoso"**

\section{Resumo}

O aumento da incidência da obesidade e cardiopatias na população idosa levanta a questão sobre as medidas antropométricas utilizadas rotineiramente nos serviços ambulatoriais do Sistema Único de Saúde (SUS), que podem estimar o risco cardiovascular (RCV). O presente estudo teve como objetivo avaliar o desempenho de dois indicadores de obesidade abdominal correlacionados como Índice de Massa Corporal (IMC): circunferência da cintura (CC) e a razão cintura/quadril (RCQ), assim como, investigar os principais fatores de risco associados em pacientes idosos atendidos na rede básica de saúde do município de Belém - PA. Foi realizado um estudo descritivo populacional em três Unidades Básicas de Saúde (UBS) no período compreendido entre junho e agosto de 2009, que incluiu 58 idosos $(66,8 \pm 5,9$ anos) escolhidos de forma consecutiva e que quiseram participar de forma voluntá- ria da pesquisa. Os dados mais relevantes na caracterização do grupo e na identificação das situações de RCV foram: baixo nível escolar $(70,6 \%)$, alimentação inadequada $(63,8 \%)$, tabagismo $(41,4 \%)$, etilismo $(51,7 \%)$ e sedentarismo $(50 \%)$. A CC e a RCQ se correlacionaram com o índice de massa corporal (IMC), sendo que a CC apresentou uma moderada correlação tanto para homens $(r=0,579 ; P<0,003)$ quanto para mulheres $(r=0,646 ; P<0,001)$, enquanto que a RCQ apresentou uma fraca correlação ( $r=0,337, \mathrm{P}<0,001$ para homens; $r=0,194, \mathrm{P}<0,04$ para mulheres). Os dados encontrados no estudo confirmam que a melhor correlação para medir o RCV na amostra de idosos estudada foi entre IMC e circunferência da cintura para ambos os gêneros.

Palavras-chave: Antropometria. Obesidade abdominal. Fatores de risco. Envelhecimento.

* Doutorando em Teoria e Pesquisa do Comportamento, Universidade Federal do Pará- UFPA, Belém, Pará, Brasil. Especialista em Gerontologia- FHCGV/UFPA. Fisioterapeuta da Secretaria Municipal de Educação de Belém e Secretaria de Estado de Saúde Pública do Pará. Endereço para correspondência: Rua Liberato de Castro, 532, Guamá, Belém-PA, Brasil. CEP: 66045-420. E-mail: rodgn@ @otmail.com

** Terapeuta Ocupacional da Caixa de Assistência dos funcionários do Banco da Amazônia- CASF, Pará.

**** Terapeuta Ocupacional residente do Programa Saúde da Família, Universidade do Estado do Pará-UEPA.

$\rightarrow$ http://dx.doi.org/10.5335/rbceh.2012.3404

Recebido em: 13/08/2013. Aprovado em: 22/08/2014 


\section{Introdução}

A população idosa no panorama internacional est crescendo de forma rápida e intensa. No Brasil, segundo o Ministério da Saúde (2011), entre 2000 e 2010, a população de idosos passou de 14 para 21 milhões.

O processo de envelhecimento caracteriza-se por perdas estruturais e funcionais, que facilitam o aparecimento de doenças crônicas interferindo sobremaneira na qualidade de vida dos longevos (PEREIRA et al., 2008). A crescente incidência das doenças cardiovasculares importante consequência do envelhecimento da população mundial, constituindo a principal causa de óbito entre os idosos (ROMERO et al., 2010).

Segundo Mendes (2012), os principais fatores determinantes do aumento das condições crônicas são as mudanças demográficas e as mudanças nos padrões de consumo e nos estilos de vida. Hábitos não saudáveis vão se estabelecendo e incrementando as condições crônicas, como tabagismo, consumo excessivo de bebidas alcoólicas, sexo inseguro, inatividade física, alimentação inadequada, estresse social e excesso de peso.

A prevalência de obesidade tem aumentado em todo o mundo e vem se tornando o maior problema de saúde na maioria dos países desenvolvidos e em desenvolvimento (ESKINAZI et al., 2011). Quando comparados aos indivíduos com peso normal, aqueles com sobrepeso apresentam maior risco de desenvolver diabetes mellitus, dislipidemia, hipertensão arterial e outras condições que favorecem o desenvolvimento de doenças cardiovasculares (CARNEIRO et al., 2003).

Diante desse cenário as doenças cardiovasculares destacam-se com estimativas alarmantes no Brasil, alcançando $31,88 \%$ das causas de óbitos, segundo o Ministério da Saúde (2011). Sabe-se que as incidências dessas doenças elevam-se com a idade, no entanto, grande parte delas pode ser evitada. Como as doenças crônicas mais prevalentes (ex. cardiovasculares, câncer e diabetes mellitus) compartilham vários fatores de risco, a Organização Mundial da Saúde (OMS) propõe uma abordagem de prevenção e controle integrado, em todas as idades e baseada na redução dos seguintes fatores: hipertensão arterial sistêmica (HAS), fumo, álcool, inatividade física, dieta inadequada, obesidade, hipercolesterolemia (PEREIRA et al., 2008).

Pesquisas mostram que esses fatores tendem a ocorrer simultaneamente (PEREIRA; BARRETO; PASSOS, 2009). A sua combinação, usualmente, representa um risco total aumentado para doenças cardiovasculares, comparado ao risco resultante da soma de seus efeitos isolados, indicando efeito sinérgico entre eles. Portanto, uma prevenção efetiva das doenças cardiovasculares s ser alcançada com a melhoria global do perfil de risco de indivíduos e populações (ROSINI et al., 2006; MONTENEGRO NETO, 2008). Por isso, a importância de se identificar riscos precocemente na Atenção Básica de Saúde e de se avaliar adequadamente os dados antropométricos da população.

Considerando que o acúmulo de gordura na região abdominal - obesidade abdominal - um importante fator de risco 
cardiovascular (RCV). Tanto na prática clínica quanto em pesquisas científicas, a antropometria vem sendo largamente utilizada para indicar o RCV por meio da avaliação de alguns indicadores, como: índice de massa corporal (IMC), circunferência da cintura (CC) e razão cintura-quadril (RCQ) (WHO, 2008; VASQUES, 2010; FRANCISCHI, 2000).

A correlação entre o RCV e diversas medidas antropométricas está bem documentada na literatura, tais como IMC, $\mathrm{CC}, \mathrm{RCQ}$, relação cintura-estatura (RCE) e o índice de conicidade (IC) (SOCIEDADE BRASILEIRA DE CARDIOLOGIA, 2010). Todavia, apesar de a maioria dos estudos já realizados com amostras de idosos apontarem as associações entre IMC-CC e IMC-RCQ como indicativos estatisticamente significativos para predição de RCV, pouco se sabe sobre qual a melhor correlação entre os indicadores antropométricos que melhor representa o RCV nessa população (ARAÚJO et al., 2011; OLIVEIRA et al., 2013; FARINEA; RICALDE; SIVIERO, 2013).

Pesquisas com a população adulta, como as de Esmaillzadeh e Mirmiran (2004) e Kragelund C, Omland (2005) sugeriram que a RCQ define melhor indivíduos de risco para doença cardiovascular. J Lemos-Santos (2004) discorda e considera que o melhor preditor de obesidade, dislipidemia e risco cardiovascular a medida da cintura (CC).

O presente estudo teve como objetivo avaliar o desempenho de dois indicadores de obesidade abdominal correlacionados como Índice de Massa Corporal (IMC): circunferência da cintura (CC) e a razão cintura/quadril (RCQ), assim como, investigar os principais fatores de risco associados em pacientes idosos atendidos na rede básica de saúde do município de Belém - PA.

\section{Material e métodos}

Trata-se de um estudo descritivo populacional com método quantitativo-exploratório em uma amostra de 58 idosos de ambos os gêneros com idade igual ou superior a 60 anos, recrutados de forma consecutiva entre aqueles em atendimento ambulatorial no Programa de Atenção à saúde do idoso de três Unidades Básicas de Saúde do município de Belém- PA, realizado no período compreendido entre junho e agosto de 2009.

Foram selecionados 62 idosos, no entanto, 6,4\% $(n=4)$ configuraram perda amostral, pois apresentavam alterações cognitivas que inviabilizaram o preenchimento dos questionários. A pesquisa foi conduzida de acordo com os princípios éticos e metodológicos contidos na Declaração de Helsinki e as regras do CNS 196/96, sendo autorizada pelos locais da pesquisa e aprovado pelo Comit de Ética da Fundação Hospital de Clínicas Gaspar Viana, sob o $\mathrm{n}^{\circ}$ 060/10. Todos os idosos que aceitaram cooperar com a realização da pesquisa formalizam a autorização no termo de consentimento livre e esclarecido (TCLE) elaborado pelos autores.

A avaliação dos pacientes seguiu um protocolo padronizado elaborado pelos autores na forma de entrevista semi-aberta incluindo três grupos de variáveis: características sociodemográficas, fatores de RCV e medidas antropométricas. 
Quanto às características sociodemográficas foram investigadas as seguintes variáveis: cor, estado civil, escolaridade, ocupação e faixa de renda mensal.

Em relação às variáveis relacionadas aos fatores de RCV foram adotados os seguintes critérios: considerou-se a presença de doenças cardiovasculares pelo auto-relato direto dos idosos ou responsáveis, pela história prévia de diagnóstico clínico registrado nos prontuários das UBS.

Foram considerados idosos hipertensos, diabéticos e dislipidêmicos os sujeitos que afirmaram serem portadores de tais morbidades durante a entrevista ou que faziam uso de medicações para controle dessas com devido registro nos prontuários durante os últimos seis meses.

A avaliação da dieta foi realizada com o auxílio do Questionário de Frequência Alimentar (QFA) adaptado de Firsberg et al. (2005). Com isso pôde-se identificar a adequação/inadequação no consumo dos grupos alimentares.

Quanto à atividade física, os idosos foram considerados sedentários quando negavam prática regular de atividade física (pelo menos, 30min por dia, três vezes/semana) (SOCIEDADE BRASILEIRA DE CARDIOLOGIA, 2010).

Considerou-se como fumante o idoso que fumava regularmente na época da inclusão no estudo em média de três ou mais cigarros por dia ou que havia parado at um ano antes.

A presença de etilismo foi definida como consumo regular de bebida alcoólica, categorizado tanto como em "beber socialmente, no máximo nos finais de semana, quanto "beber excessivamente, beber todos os dias ou quando começa a beber não consegue parar.

Em relação ao uso de medicamentos, foram considerados usuários os idosos que administravam pelo menos um medicamento diário, considerando as vias oral, retal e parentérica.

Os indicadores antropométricos coletados como variáveis independentes foram: índice de massa corporal $\left(\mathrm{kg} / \mathrm{m}^{2}\right)$, circunferência da cintura (CC), razão cintura-quadril (RCQ). A massa corporal foi medida em uma balança digital portátil da marca Soehnle Professional 7755 , com precisão de $100 \mathrm{~g}$ e a estatura foi medida por um estadiômetro da mesma marca, com precisão de $0,1 \mathrm{~cm}$. $\mathrm{O}$ IMC foi calculado pelo quociente entre a massa e o quadrado da estatura. Foram considerados obesos os indivíduos com IMC $\geq 30 \mathrm{~kg} / \mathrm{m} 2$ (LEE; NIEMAN, 1996).

As circunferências da cintura (na altura da cicatriz umbilical) e do quadril (porção de maior diâmetro) foram coletadas com uma fita métrica da marca Cardiomed $^{\circledR}$ com precisão de $1 \mathrm{~mm}$. A avaliação da RCQ se sucedeu conforme critério da OMS. Foi obtida dividindo-se a circunferência da cintura pela do quadril, ambas em centímetros $(\mathrm{cm}) \mathrm{e}$ considerou-se elevados os valores acima de 0,8 para mulheres e 0,95 para os homens (WHO, 2008).

A pressão arterial foi medida com esfigmomanômetro e estetoscópio da marca Premium ${ }^{\circledR}$ pelo método auscultatório, duas vezes, no braço esquerdo, após 10 minutos de repouso na posição sentada. Hipertensão arterial sistêmica (HAS) foi definida pelas médias pressóricas $\geq 140 / 90 \mathrm{mmHg}$ ou pelo uso de medicações 
anti-hipertensivas (SOCIEDADE BRASILEIRA DE CARDIOLOGIA, 2002).

Para a análise dos dados utilizou-se os programas Epi Info 3.5.2 (Centers for Disease Control and Prevention, Atlanta, Estados Unidos) e BioEstat 5.0 (Sociedade Civil Mamirau, Manaus, Brasil). A análise descritiva dos dados serviu para caracterizar a amostra, com a distribuição de frequência e seus respectivos intervalos de confiança (IC95\%). Além disso, foi usado também o teste de correlação de Spearman para se testar a correlação entre as medidas da CC e RCQ com os valores do IMC, massa, pressão sistólica e pressão diastólica.

\section{Resultados}

Um total de 58 pacientes idosos (24 homens, 34 mulheres) foi incluído no estudo. Os pacientes apresentaram 66,8 $\pm 5,9$ anos de idade. A tabela 1 apresenta as variáveis envolvendo características sociodemográficas. A análise esclareceu que $51,7 \%$ dos idosos eram de cor branca, $55,1 \%$ casados, $70,6 \%$ apresentaram nível de escolaridade at sete anos de estudo, $63,7 \%$ eram economicamente inativos e $63,8 \%$ com renda per capita de at um salário mínimo mensal.

Tabela 1: Distribuição dos idosos, segundo as características sociodemográficas e gênero. Belém, PA, 2009

\begin{tabular}{|c|c|c|c|c|c|c|c|c|c|}
\hline \multirow{2}{*}{ Variáveis } & \multicolumn{3}{|c|}{ Masculino } & \multicolumn{3}{|c|}{ Feminino } & \multicolumn{3}{|c|}{ Total } \\
\hline & $\mathrm{N}$ & $\%$ & IC95\% & $\mathrm{N}$ & $\%$ & IC95\% & $\mathrm{N}$ & $\%$ & IC95\% \\
\hline \multicolumn{10}{|l|}{ Cor } \\
\hline Branca & 10 & 41,6 & $22.1-63.4$ & 20 & 58,8 & $24.6-59.3$ & 30 & 51,7 & $34.6-59.2$ \\
\hline Não branca & 14 & 58,3 & $36.6-77.9$ & 14 & 41,1 & $40.7-75.4$ & 28 & 48,2 & $39.5-62.3$ \\
\hline \multicolumn{10}{|l|}{ Estado civil } \\
\hline Casado & 20 & 83,3 & $62.6-95.3$ & 12 & 35,3 & $19.7-53.5$ & 32 & 55,2 & $41.5-68.3$ \\
\hline Solteiro & 4 & 16,7 & $4.7-37.4$ & 10 & 29,4 & $15.1-47.5$ & 14 & 24,1 & 13.9-37.2 \\
\hline Viúvo & -- & -- & -- & 11 & 32,4 & $17.4-50.5$ & 11 & 19,0 & $9.9-31.4$ \\
\hline Separado & -- & -- & -- & 1 & 2,9 & 0.1-15.3 & 1 & 1,7 & $0.0-9.2$ \\
\hline \multicolumn{10}{|l|}{ Escolaridade } \\
\hline Analfabeto & 4 & 16,7 & $4.7-37.4$ & 2 & 5,9 & $0.7-19.7$ & 6 & 10,0 & $3.9-21.2$ \\
\hline At 7 anos & 13 & 54,2 & $32.8-74.4$ & 28 & 82,4 & $65.5-93.2$ & 41 & 71,0 & $57.3-81.9$ \\
\hline Acima 7 anos & 7 & 29,2 & 12.6-51.1 & 4 & 11,8 & 3.3-27.5 & 11 & 19,0 & $9.9-31.4$ \\
\hline \multicolumn{10}{|l|}{ Ocupação } \\
\hline Inativos & 12 & 50,0 & 29.1-70.9 & 9 & 26,5 & $12.9-44.4$ & 37 & 63,8 & $50.1-76.0$ \\
\hline Ativos & 12 & 50,0 & 29.1-70.9 & 25 & 73,5 & $55.6-87.1$ & 21 & 36,2 & $24.0-49.9$ \\
\hline \multicolumn{10}{|l|}{ Renda mensal } \\
\hline At 1 salário mínimo & 11 & 45,8 & $25.6-67.2$ & 26 & 76,4 & $58.8-89.3$ & 37 & 63,8 & $50.1-76.0$ \\
\hline 1 a 3 salários mínimos & 10 & 41,7 & 22.1-63.4 & 8 & 23,5 & $10.7-41.2$ & 18 & 31,0 & $19.5-44.5$ \\
\hline 4 a 6 salários mínimos & 1 & 4,2 & $0.1-21.1$ & -- & -- & -- & 1 & 1,7 & $0.0-9.2$ \\
\hline $\begin{array}{l}\text { Mais de } 7 \text { salários } \\
\text { mínimos }\end{array}$ & 2 & 8,3 & $1.0-27.0$ & -- & -- & -- & 2 & 3,4 & $0.4-11.9$ \\
\hline
\end{tabular}


A Tabela 2 demonstra a distribuição das variáveis relacionadas aos fatores de RCV com os respectivos IC $95 \%$.

Tabela 2: Distribuição dos idosos, segundo fatores de RCV e gênero. Belém, PA, 2009

\begin{tabular}{|c|c|c|c|c|c|c|c|c|c|}
\hline \multirow{2}{*}{ Variáveis } & \multicolumn{3}{|c|}{ Masculino } & \multicolumn{3}{|c|}{ Feminino } & \multicolumn{3}{|c|}{ Total } \\
\hline & $\mathrm{N}$ & $\%$ & IC95\% & $\mathrm{N}$ & $\%$ & IC95\% & $\mathrm{N}$ & $\%$ & $\mathrm{IC} 95 \%$ \\
\hline \multicolumn{10}{|c|}{ Doenças cardiovasculares } \\
\hline Presente & 10 & 41,7 & $22.1-63.4$ & 12 & 35,3 & $19.7-53.5$ & 22 & 37,9 & $25.5-51.6$ \\
\hline Ausente & 14 & 58,3 & $36.6-77.9$ & 22 & 64,7 & $46.5-80.3$ & 36 & 62,1 & $48.4-74.5$ \\
\hline \multicolumn{10}{|l|}{ HAS } \\
\hline Presente & 15 & 62,5 & $40.6-81.2$ & 24 & 70,6 & $52.5-84.9$ & 39 & 67,2 & $53.7-79.0$ \\
\hline Ausente & 9 & 37,5 & $18.8-59.4$ & 10 & 29,4 & $15.1-47.5$ & 19 & 32,8 & $21.0-46.3$ \\
\hline \multicolumn{10}{|l|}{ Diabetes } \\
\hline Presente & 12 & 50,0 & $29.1-70.9$ & 11 & 32,35 & $17.4-50.5$ & 23 & 39,7 & $27.0-53.4$ \\
\hline Ausente & 12 & 50,0 & $29.1-70.9$ & 23 & 67,6 & $49.5-82.6$ & 35 & 60,3 & $46.6-73.0$ \\
\hline \multicolumn{10}{|l|}{ Dislipidemia } \\
\hline Presente & 14 & 58,3 & $36.6-77.9$ & 14 & 41,2 & 24.6-59.3 & 28 & 48,3 & $35.0-61.8$ \\
\hline Ausente & 10 & 41,7 & 22.1-63.4 & 20 & 58,8 & $40.7-75.4$ & 30 & 51,7 & $38.2-65.0$ \\
\hline \multicolumn{10}{|l|}{ Dieta } \\
\hline Inadequada & 10 & 41,7 & $22.1-63.4$ & 27 & 79,4 & $62.1-91.3$ & 37 & 63,8 & $50.1-76.0$ \\
\hline Adequada & 14 & 58,3 & $36.6-77.9$ & 7 & 20,6 & 8.7-37.9 & 21 & 36,2 & $24.0-49.9$ \\
\hline \multicolumn{10}{|l|}{ Atividade física } \\
\hline Ativos & 10 & 41,7 & $22.1-63.4$ & 19 & 55,9 & $37.9-72.8$ & 29 & 50,0 & $36.6-63.4$ \\
\hline Sedentários & 14 & 58,3 & $36.6-77.9$ & 15 & 44,1 & $27.2-62.1$ & 29 & 50,0 & $36.6-63.4$ \\
\hline \multicolumn{10}{|l|}{ Tabagismo } \\
\hline Fumantes & 11 & 45,8 & $25.6-67.2$ & 13 & 38,2 & $22.2-56.4$ & 24 & 41,4 & $28.6-55.1$ \\
\hline Não fumantes & 13 & 54,2 & $32.8-74.4$ & 21 & 61,8 & 43.3-77.8 & 34 & 58,6 & $44.9-71.4$ \\
\hline \multicolumn{10}{|l|}{ Etilismo } \\
\hline Presente & 15 & 62,5 & $40.6-81.2$ & 15 & 44,1 & $27.2-62.1$ & 30 & 51,7 & $38.2-65.0$ \\
\hline Ausente & 9 & 37,5 & 18.8-59.4 & 19 & 55,9 & $37.9-72.8$ & 28 & 48,3 & $35.0-61.8$ \\
\hline \multicolumn{10}{|c|}{ Uso de medicamentos } \\
\hline Usuário & 22 & 91,7 & $73.0-99.0$ & 29 & 85,3 & $68.9-95.0$ & 51 & 87,9 & $76.7-95.0$ \\
\hline Não usuário & 2 & 8,3 & $1.0-27.0$ & 5 & 14,7 & $5.0-31.1$ & 7 & 12,1 & $5.0-23.3$ \\
\hline
\end{tabular}


Na Tabela 3 estão descritas as correlações entre a RCQ e a medida da cintura com variáveis que estimam fatores de RCV (IMC, massa, PAS e PAD). A medida da cintura e a RCQ se correlacionaram com o IMC, sendo que a cintura apresentou uma moderada correlação, tanto para homens $(\mathrm{r}=0,579$;
$\mathrm{P}<0,003)$ quanto para mulheres $(\mathrm{r}=$ 0,$646 ; \mathrm{P}<0,001$ ), enquanto que a RCQ apresentou uma fraca correlação $(\mathrm{r}=$ $0,337, \mathrm{P}<0,001$ para homens; $r=0,194$, $\mathrm{P}<0,04$ para mulheres). A mesma correlação foi obtida para a massa. Sobre as medidas de PA não foram observadas correlações importantes dos dados.

Tabela 3: Correlação entre a razão cintura-quadril e a medida da circunferência da cintura com as variáveis IMC $(\mathrm{kg} / \mathrm{m} 2)$, Massa $(\mathrm{kg})$, Pressão sistólica $(\mathrm{mmHg})$ e Pressão diastólica $(\mathrm{mmHg})$

\begin{tabular}{l|r|r|r|r}
\cline { 2 - 5 } & \multicolumn{2}{c|}{ Homens } & \multicolumn{2}{c}{ Mulheres } \\
\cline { 2 - 5 } & \multicolumn{1}{c}{ RCQ } & \multicolumn{1}{c|}{ Cintura } & \multicolumn{1}{c}{ RCQ } & \multicolumn{1}{c}{ Cintura } \\
\hline IMC $(\mathrm{kg} / \mathrm{m} 2)$ & 0.337 & 0.579 & 0.194 & 0.646 \\
Massa $(\mathrm{kg})$ & 0.334 & 0.596 & 0.204 & 0.643 \\
Pressão sistólica $(\mathrm{mmHg})$ & -0.032 & 0.180 & 0.059 & 0.107 \\
Pressão diastólica $(\mathrm{mmHg})$ & -0.167 & 0.180 & 0.131 & 0.222 \\
\hline
\end{tabular}

\section{Discussão}

O inquérito realizado nos forneceu dados importantes sobre os fatores de RCV da população idosa estudada. Apesar da heterogeneidade do grupo de idosos estudado, alguns dados merecem destaque e servem como base estatística para direcionamento dos serviços terapêuticos e principalmente dos serviços preventivos, que podem ser desenvolvidos orientando a modificação de hábitos e estilo de vida. Pereira et al. (2008) afirma em seu estudo que as populações envelhecidas são heterogêneas, assim como a da nossa pesquisa, e a análise de fatores de riscos difere daquela para adultos mais jovens. Suas características são influenciadas, sobretudo, por eventos históricos que marcam as diferentes coortes de nascimento, viés de sobrevivência e por diferenças entre gêneros.

Diante do exposto nos resultados, observou-se que a maioria dos idosos estudados $(70,6 \%)$ era de baixo nível escolar e econômico. Essas informações refletem os mesmos dados encontrados na maioria dos estudos com essa faixa etária, incluindo os de Vicentini et al. (2008) e Pereira et al. (2008).

Os dados mais preocupantes do estudo envolvem os relacionados ao estilo de vida. Nesses destacam-se a alimentação inadequada $(63,8 \%)$, o tabagismo $(41,4 \%)$, o etilismo $(51,7 \%)$ e o sedentarismo (50\%). Assim como no estudo de Pereira et al. (2008), foi observado no estudo uma maior proporção de idosos que mantém hábitos alimentares inadequados. Isso demonstra a importância 
do nutricionista na atenção básica atuando de forma direta nos programas de atenção ao idoso para modificar hábitos alimentares errôneos. No entanto, a ausência do acompanhamento nutricional inviabiliza o tratamento dietético individualizado, essencial para melhor adesão do paciente e eficácia da terapêutica não medicamentosa.

Com relação ao tabagismo, o dado encontrado foi muito parecido com os dados de Mendonça (2004). Homens também são mais prevalentes e pode-se explicar isso por aspectos culturais e históricos. A redução das prevalências de fatores de risco como tabagismo e consumo de álcool com o envelhecimento pode refletir tanto a mortalidade precoce de adultos mais jovens expostos a esses fatores, quanto mudanças de comportamentos de risco após adoecimento, principalmente em idosos mais frágeis.

O número reduzido de idosos fisicamente ativos pode ser decorrência, em parte, da existência de barreiras como a falta de orientação e de locais adequados para realização de exercícios. A atividade física, além de promover interação social entre idosos, preserva a independência nas atividades da vida diária e contribui para redução e o controle de fatores de RCV e de condições como as doenças coronarianas. A prevalência de inatividade física foi muito superior aos resultados encontrados por Mendonça (2004) e Forjaz et al. (2002). Segundo Matsudo et al. (2001), a prevalência de inatividade aumenta com a idade, podendo atingir valores acima de $70 \%$.

Segundo Furtado e Polanczyk (2007), as modificações no estilo de vida, como dieta pobre em carboidratos, redução de peso e prática regular de atividade física, devem ser instituídas. Ainda devem manter o controle metabólico de lipídios e da glicemia e controlar a pressão arterial, visando uma melhor qualidade de vida.

Assim como em outros estudos como, por exemplo, de Mendonça et al. (2004), Rosini et al. (2006) e Carolino et al. (2008), os dados referentes HAS foram os de maior prevalência $(67,2)$. Sabe-se que a HAS mais prevalente entre os idosos e aumenta sua proporção com o avanço da idade.

Com relaão aos dados referentes correlação IMC-CC e IMC-RCQ em idosos, observou-se no presente estudo que a correlação em ambos os gêneros foi estatisticamente significante; entretanto, não foi tão forte como a correlação identificada com a circunferência da cintura o que corrobora com resultados encontrados em outros estudos descritos logo abaixo.

Dobbelsteyn et al. (2001) avaliaram 500 homens e 501 mulheres chinesas e identificaram a CC como medida preditiva de RCV, especialmente em mulheres, seguida da RCQ, enquanto que para os homens ressaltou-se o IMC e CC. Goodman-Gruen e Barret-Connor (1996), Zamboni et al. (1998) e Armellini et al. (1994) demonstraram correlações parecidas quando compararam IMC e $\mathrm{RCQ} / \mathrm{CC}$ em mulheres idosas.

Sampaio e Figueiredo (2005) também obtiveram resultados parecidos quando correlacionaram os mesmos dados em adultos e idosos. Elas concluíram que tanto no grupo dos adultos quanto no dos idosos, de ambos os gêneros, a correlação entre o IMC e a CC foi elevada. 
Segundo Pitanga (2007), essa relação se deve associação da gordura abdominal com diversas alterações metabólicas como, diabetes, hipertensão, intolerância a glicose e alterações nas concentrações de lipoproteínas, que frequentemente são encontradas nas doenças cardiovasculares.

Essas alterações metabólicas correlacionaram-se com a maioria dos fatores de risco cardiovascular, principalmente com níveis elevados de triglicérides e reduzidos de HDL, apresentando maior impacto sobre a elevação da pressão arterial, como visto marcadamente na literatura (CARNEIRO et al., 2003).

Em estudos realizados por Rezende et al. (2006) concluiu-se que indivíduos com excesso de peso, principalmente com obesidade abdominal, estão mais expostos a fatores de risco cardiovasculares envolvidos na síndrome metabólica e, consequentemente, a maior risco de morbidade e mortalidade quando essas alterações não são tratadas. Fatores de risco como sedentarismo, sobrepeso/obesidade, distribuição central da gordura corporal, tabagismo e consumo excessivo de bebidas alcoólicas são passíveis de intervenção.

Os dados encontrados no estudo confirmam que a melhor correlação para medir o RCV em idosos é entre IMC e circunferência da cintura em ambos os gêneros. Quanto a alguns aspectos de estilo de vida, observaram-se elevadas proporções relacionadas ao hábito do tabagismo, etilismo, inatividade física e maus hábitos alimentares pela maioria dos idosos pesquisados nas três unidades municipais de saúde.
Embora alguns dados científicos apontem para as mesmas conclusões com relação ao melhor parâmetro utilizado para medir o RCV, ainda são necessários estudos prospectivos com número maior de idosos para se definir na prática ambulatorial os melhores métodos de avaliação e acompanhamento dos mesmos.

\section{Performance of abdominal obesity and cardiovascular risk indicators of elderly assisted in primary health care in Belém - PA}

\section{Abstract}

The increased incidence of obesity and heart diseases in the elderly population raises a question concerned the anthropometric measurements used routinely in the outpatient services of the public health system (SUS) that can estimate the cardiovascular risk. This study aims to evaluate the performance of two measures of abdominal obesity correlated as Body Mass Index (BMI): Waist Measure (WM) and Waist-to-Hip Ratio (WHR) in discriminating risk of development of cardiovascular disease and to investigate the cardiovascular risk behaviour in elderly patients treated in basic health network of the city of Belém-PA. A descriptive population-based study was carried out in three Basic Health Units (BHU) from June to August 2009 that included 58 elderly $(66,8 \pm$ 5.9 years), selected consecutively. The most relevant data on the characterization of the group and in the identification of situations of cardiovascular risk were: low education $(70.6 \%)$, inadequate nutrition $(63.8 \%)$, smoking (41.4\%), alcoholism (51.7\%) and sedentarism (50\%). The WM and the WHR was correlated with the body mass index $(\mathrm{BMI})$, the WM presented a moderate correlation for men $(r=0.579 ; P<0.003)$ and women $(r=0.646 ; P<0.001)$. On the other 
hand, the WHR was weakly correlated $(r=$ $0.337, \mathrm{P}<0.001$ for men; $\mathrm{r}=0.194, \mathrm{P}<0.04$ for women). The data found in the study confirm that the best correlation to measure cardiovascular risk in elderly sample studied was between BMI and WM for both genders.

Keywords: Anthropometry. Obesity abdominal. Risk factors. Aging.

\section{Referências}

ARAÚJO, L. G. B de. et al. Perfil nutricional de adultos e idosos atendidos na rede municipal de saúde de Manaus e sua associação a doenças crônico-degenerativas. RBCEH, Passo Fundo, v. 8, n. 1, p. 59-69, jan./abr. 2011.

ARMELLINI, F. et al. Measured and predicted total and visceral adipose tissue in women. Correlations with metabolic parameters. Int $J$ Obes Relat Metab Disord., London, v. 18, n. 9, p. 641-647, maio 1994.

BRASIL. Ministério da Saúde. DATASUS. Informações de Saúde. Estatísticas vitais - Mortalidade e nascidos vivos. 2007. Disponível em: <http://tabnet.datasus.gov.br>. Acesso em: 21 jan. 2013.

CARNEIRO, G. et al. Influência da distribuição da gordura corporal sobre a prevalência de hipertensão arterial e outros fatores de risco cardiovascular em indivíduos obesos. Rev Assoc Med Bras, São Paulo, v. 49, n. 3, p. 306-311, jul./set. 2003.

CAROLINO, I. D. R. et al. Fatores de risco em pacientes com diabetes mellitus tipo 2. Rev. Latino-Am. Enfermagem (online), Ribeirão Preto, v. 16, n. 2, p. 238-244, mar./ abr. 2008.

DOBBELSTEYN, C. J. et al. A comparative evaluation of waist circumference, waist-tohip ratio and body mass index as indicators of cardiovascular risk factors. The Canadian Heart Health Surveys. Int J Obes., v. 25, n. 5, p. 652-661, nov./dez. 2001.
ESKINAZIA, F. M. V. et al. Envelhecimento e a Epidemia da Obesidade. UNOPAR, Cient Ciênc Biol Saúde, Paraná, v. 13(Esp), p. 295298, dez. 2011.

ESMAILLZADEH, A.; MIRMIRAN, P.; AZIZI, F. Waist-to-hip ratio is a better screening measure for cardiovascular risk factors than other anthropometric indicators in Tehranian adult men. Int J Obes Relat Metab Disord, London, v. 28, n. 10, p. 1325-1332, maio 2004

FISBERG, M. R. et al. Inquéritos alimentares: métodos e bases científicos. São Paulo: Manole; 2005.

FORJAZ, C. L. M. et al. Avaliação do risco cardiovascular e da atividade física dos frequentadores de um parque da cidade de São Paulo. Arq Bras Cardiol., Rio de Janeiro, v. 79, n. 1, p. 35-50, jul. 2002.

FARINEA N.; RICALDE S. R.; SIVIERO, J. Perfil nutricional e antropométrico de idosos participantes de um grupo de ginástica no município de Antônio Prado- RS. RBCEH, Passo Fundo, v. 7, n. 3, p. 394-405, set./dez. 2010.

FRANCISCHI, R. P. P. et al. Obesidade: atualização sobre sua etiologia, morbidade e tratamento. Revista de Nutrição, Campinas, v. 13 , n. 1 , p. 17-28, jan./abr. 2000.

FURTADO, M. V.; POLANCZYK, C. A. Cardiovascular prevention in diabetic patients: an evidenced-based review. Arq Bras Endocrinol Metab., São Paulo, v. 51, n. 2, p. 312-318, mar. 2007.

GOODMAN-GRUEN, D.; BARRET-CONNOR, E. Sex differences in measures of body fat and body fat distribution in the elderly. Am J Epidemiol., Baltimore, v. 143, n. 9, p. 898-906, maio 1996.

INSTITUTO BRASILEIRO DE GEOGRAFIA E ESTATÍSTICA (IBGE). Anuário Estatístico do Brasil. Brasília: IBGE; 2004. 
KANNEL, W. B.; WILSON, P. W. Comparison of risk profiles for cardiovascular events: implications for prevention. Arch Intern Med., San Francisco, n. 42, p. 39-66, Jan. 1997.

LEE, R. D.; NIEMAN, D. C. Nutritional Assessment, 2. ed. St Louis, Mosby, 1996.

LEMOS-SANTOS, M. G. et al. Waist circumference and waist-to-hip ratio as predictors of serum concentration of lipids in Brazilian men. Nutrition, London, v. 20, n. 10, p. 857862, Oct. 2004.

MATSUDO, S. M. M. Evolução da aptidão física e capacidade funcional de mulheres ativas acima de 50 anos de idade de acordo com a idade cronológica. São Paulo, 2001. 266 f. Tese (Doutorado em Ciências) - Escola Paulista de Medicina, Universidade Federal de São Paulo, 2001.

MENDONÇA, T. T. et al. Risco cardiovascular, aptidão física e prática de atividade física de idosos de um parque de São Paulo. Revista brasileira Ciência e Movimento, Distrito Federal, v. 12 n. 2, p. 19-24, jun. 2004.

MONTENEGRO NETO, A. N. Obesidade, envelhecimento e risco cardiovascular no Brasil: possíveis soluções para problemas atuais. Rev. Saúde. Com., Bahia, v. 4, n. 1, p. 57-63, jan./jun. 2008.

OLIVEIRA, L. M. et al. Associação entre obesidade geral e abdominal com a hipertensão arterial em idosas ativas. Rev. Educ. Fis / UEM, Maring, v. 24, n. 4, p. 659-668, out./ dez. 2013.

PEREIRA, J. C.; BARRETO, S. M.; PASSOS, V. M. Perfil de Saúde Cardiovascular dos idosos Brasileiros Precisa melhorar: Estudo de Base Populacional. Arq Bras Cardiol., Rio de Janeiro, v. 91, n. 1, p. 1-10, jul. 2008.

PITANGA, F. J. G.; LESSA, I. Associação entre indicadores antropométricos de obesidade e risco coronariano em adultos. Rev Bras Epidemiol., São Paulo, v. 10, n. 2, p. 239-248, jun. 2007.
REZENDE, F. A. C. et al. Índice de Massa Corporal e Circunferência Abdominal: Associação com Fatores de Risco Cardiovascular. Arq Bras Cardiol., Rio de Janeiro, v. 87, n. 6, p. 728-734, dez. 2006.

ROMERO, A. D. et al. Características de uma população de idosos hipertensos atendida numa unidade da família. Rev. Rene, v. 11, n. 2, p. 72-78, abr./jun. 2010.

ROSINI, N.; MACHADO, M. J.; XAVIER, H. T. Estudo de Prevalência e Multiplicidade de Fatores de Risco Cardiovascular em Hipertensos do Município de Brusque, SC. Arquivos Brasileiros de Cardiologia, Rio de Janeiro, v. 86, n. 3, p. 219-222, mar. 2006.

SAMPAIO, L. R.; FIGUEIREDO, V. C. Correlação entre o índice de massa corporal e os indicadores antropométricos de distribuição de gordura corporal em adultos e idosos. Rev. Nutrição, Campinas, v. 18, n. 1, p. 53-61, jan./fev. 2005.

SOCIEDADE BRASILEIRA DE CARDIOLOGIA; SOCIEDADE BRASILEIRA DE HIPERTENSÃO; SOCIEDADE BRASILEIRA DE NEFROLOGIA. IV Diretrizes Brasileiras de Hipertensão Arterial. Revista Hipertensão, São Paulo, v. 5, n. 4, p. 126-163, fev. 2002.

VI Diretrizes Brasileiras de Hipertensão Arterial. Arquivos Brasileiros de Cardiologia. São Paulo, v. 95, supl. 1, p. 1-51, jan./fev./mar. 2010.

KRAGELUND, C.; OMLAND, T. A farewell to body-mass index? Lancet; New York, v. 366 , n. 9497 , p. 1589-1591, Nov. 2005.

MENDES, E. V. O cuidado das condições crônicas na atenção primária saúde: o imperativo da consolidação da estratégia da saúde da família. Brasília: Organização Pan-Americana da Saúde; 2012.

PEREIRA, J. C; BARRETO, S. M.; PASSOS, V. M. A. Perfil de risco cardiovascular e autoavaliação da saúde no Brasil: estudo de base populacional. Rev Panam Salud Publica, Washington, v. 25, n. 6, p. 491-498, jun. 2009. 
VASQUES, A. C. J. et al. Utilização de medidas antropométricas para a avaliação do acúmulo de gordura visceral. Rev. Nutr., Campinas, v. 23, n. 1, p. 107-118, jan./fev. 2010.

VICENTINI, G. E. et al. Prevalência de fatores de risco cardiovascular em diabéticos tipo 2 no Município de Paranava-PR. Arq. Ciênc. Saúde Unipar, Umuarama, v. 12, n. 2, p. 109-117, maio/ago. 2008.

WORLD HEALTH ORGANIZATION. Waist Circumference and Waist-Hip Ratio: Report of a WHO Expert Consultation. Geneva 8-11 Dez. 2008.

ZOMBONI, M. et al. Sagittal abdominal diameter as a practical predictor of visceral fat. Int J Obes Relat Metab Disord., London, v. 22, n. 7, p. 655-660, July. 1998. 